\title{
Usability and Acceptability of JAGA SEHAT: Mobile Application to Improve Knowledge About Healthy Lifestyle
}

\author{
Aulia Iskandarsyah (1D ${ }^{1,2}$, Arina Shabrina (1D', Ahmad Gimmy Pratama Siswadi ${ }^{1,2}$ \\ 'Faculty of Psychology, Universitas Padjadjaran, Bandung, Indonesia; ${ }^{2}$ Center for Psychological Innovation and Research, Universitas Padjadjaran, \\ Bandung, Indonesia \\ Correspondence: Aulia Iskandarsyah, Faculty of Psychology, Universitas Padjadjaran, Jl. Raya Bandung Sumedang KM 21 , Jatinangor, 45363, West Java, \\ Indonesia, Tel +62 8I I2106260, Fax +62 2287920376, Email a.iskandarsyah@unpad.ac.id
}

Purpose: The use of mHealth has great potential to overcome many barriers in healthcare and become powerful tools to promote health. We developed Jaga Sehat ("Stay Healthy"), an Indonesian version of the mHealth app which was created as a form of health education for the general population. The current study aimed to test the usability and acceptability of the Jaga Sehat mHealth app. Patients and Methods: A total of 113 dentistry students who participated in the first-year initiation program at a state university in West Java, Indonesia were recruited. Participants were asked to download, use the app and complete an online questionnaire. Open response questions explored participants' recommendations for future improvement. Descriptive statistics were used to analyze participants responses, and content analysis was carried out to analyze open-ended responses.

Results: In general, participants perceived that Jaga Sehat mHealth app was useful, well-designed app, functional, and easy to use. We found that most of participants gave positive feedback and considered it as having high usability and acceptability.

Conclusion: Participants reported that Jaga Sehat mHealth app was functional, easy to use and have a good design. The language and material were clear and easy to understand. The app could encourage and help them lead a healthy lifestyle; therefore, they would recommend this app for others.

Keywords: health promotion, knowledge, internet, mobile application

\section{Introduction}

Technology has an important role in dealing with many health priorities as it relates to promotion, prevention, diagnosis, and treatment of the symptoms of the disease. The rapid growth of the internet and the spread of mobile technologies raise the potential to incorporate everyday use of technology with healthcare. The innovative application of mobile technology that focuses to address various health-related issues has evolved into a new field, known as mHealth. The WHO defined mHealth as

medical and public health practices supported by mobile devices, such as mobile phones, patient monitoring devices, personal digital assistants, and other wireless devices. ${ }^{1}$

The mHealth can transform how health information and healthcare are accessed, delivered, and managed.

Many technologies are now designed specifically to facilitate medical and health needs, contributing to the emergence of mobile digital devices, mobile applications, website, and platform which offer not only readily accessible medical and health information but also innovative ways to monitor personal health and share the information with others. ${ }^{2}$ The use of mHealth app has the potential to efficiently and effectively modify health behavior and important risk factors for various diseases among young people. ${ }^{3}$ Previous research showed that mHealth app in one respect can lead to better routine and easier living or help maintaining health lifestyle for people with chronic illness. ${ }^{4}$ The utilization of mHealth applications has shown exceptional promise as an important communication medium for supporting the use of the traditional medium 
for health promotion. ${ }^{5}$ For example, during the COVID-19 pandemic, many traditional face-to-face health consultations were rapidly changed into teleconsultations. Telemedicine and eHealth can be adopted in times of health emergency to support the treatment of patients during and after the pandemic as safer, convenient, and in line with lockdown and social distancing measure. ${ }^{6,7}$

The market for mobile health has been growing constantly from year to year. In 2016, there were approximately 259,000 mHealth apps are available globally. ${ }^{8}$ The newer report from 2017 estimated 325,000 health apps (health and fitness and medical apps) available on all major apps stores. ${ }^{9}$ mHealth app downloads were expected to reach almost 6 billion in 2020, and the number of app users was predicted to increase up to 4.5 billion users in $2020 .{ }^{10}$ With the rising popularity of mHealth, there is also a need to provide an evidence base to maximize its usefulness for the greater good and support the clinical use. ${ }^{11}$

Indonesia is the world's fourth country with the highest number of internet users. The Indonesian Internet Service Providers Association (APJII) revealed that the number of internet users in Indonesia has increased rapidly since the early development of the internet in the 1990s. Based on the APJII report from the year 2016 to 2018, the number of internet users reached 132.7 million people in 2016 (51,8\% compared to the total population of 256.2 million), 143.26 million people in 2017 (54,7\% compared to the total population of 262 million) and 171.17 million people in 2018 (64,8\% compared to the total population of 264.16 million). ${ }^{12-14}$ The number is expected to increase continuously in the following years. The 2019 survey report titled "Digital 2020: Indonesia" showed that active internet users in Indonesia reached already 175.4 million from a total population of 272.1 million. ${ }^{15}$ More than half of the Indonesian population $(64,4 \%)$ are active internet users. We perceived a huge opportunity of mHealth utilization to provide an effective, easy to use dan cost effective form of health promotion program.

Regarding eHealth's transformative potential, there are outstanding opportunities to improve the existing weak or underdeveloped healthcare systems and to address diverse health priorities among developing countries. ${ }^{16}$ In Indonesia, some mHealth apps have been developed and previous researches have been conducted to explore the process of developing a mHealth app, testing usability and feasibility, review and propose a design for the app, assess knowledge, attitudes, and behavior on utilizing the mHealth app, and factors affecting user's intention and success for mobile health implementation. The applications that are tested and reviewed were a mobile app for data monitoring in integrated healthcare center ("Pos Pelayanan Kesehatan Terpadu"), ${ }^{17}$ mHealth for improving early life nutrition care for mother and child health, ${ }^{18}$ the app that features a doctor's consultation, laboratory check, and pharmacy, ${ }^{19}$ mobile app to improve the knowledge and wellness of autoimmune sufferers ${ }^{20}$ and TB patients ${ }^{21,22}$ and mobile health app for government-based healthcare insurance. ${ }^{23}$

As mentioned above, mHealth apps that have been developed and tested in Indonesia mainly focused on managing an illness or providing a health service to users. To the best of our knowledge, we found only limited number of mHealth app that have a strong scientific and include a comprehensive information on physical and psychological aspect of health. Therefore, we developed Jaga Sehat ("Stay Healthy"), an Indonesian version of the mHealth app which was created as a form of health education for the general population. The current study aimed to test the usability and acceptability of the Jaga Sehat mHealth app.

\section{Materials and Methods}

\section{The Intervention}

Jaga Sehat is a mobile application designed as a media platform to increase the general public's knowledge about healthy living and encourage them to apply a healthy lifestyle in everyday life. Two of the authors wrote the blue print and story line (AI and AG) and developed the Mobile Apps with a collaborative expert team, including one visual communication designer/Illustrator, and one app developer. The target user for this app for the general adult population, the application was created it in the setting of a dialogue between two college students and a health psychologist in the form of cartoon illustration using an android operating system. The material was interactive (user can use the navigation buttons) and delivered through written form coupled with voice over of each character. The content of Jaga Sehat is presented in Table 1. 
Table I Jaga Sehat mHealth App Blueprint

\begin{tabular}{|c|c|}
\hline Title & JAGA SEHAT \\
\hline Format & Android Mobile Apps \\
\hline Language & Bahasa Indonesia \\
\hline $\begin{array}{l}\text { Target } \\
\text { Population }\end{array}$ & General adult population \\
\hline Objective & To increase the general public's knowledge about healthy living and encourage them to apply a healthy lifestyle in everyday life. \\
\hline Part I & Character Introduction \\
\hline Part 2 & Definition of Health and Reason to be healthy \\
\hline Part 3 & Tips for a healthy lifestyle: (a) healthy diet, (b) active lifestyle, (c) positive mindset and (d) religion/spirituality \\
\hline Part 4 & Common symptoms of illness \\
\hline Part 5 & Sources of health information and health referral center. \\
\hline
\end{tabular}

\section{Setting and Participants}

We selected faculty of Dentistry at Universitas Padjadjaran for this study setting as this faculty has an integrated the firstyear initiation program on several topics, such as academic adjustment and healthy life style. Moreover, the context of education and information was dialog between a health psychologist and college students. The convenience sampling technique was used. We approached students at the Faculty of Dentistry in 2018 who followed the first-year initiation program. One hundred forty students were invited to participate in this study, and 113 students were willing to participate voluntarily. Participants were eligible if they had Android Smartphone and agreed to participate.

\section{Procedures}

This study was reviewed and approved by the Directorate of Research and Community Services of Universitas Padjadjaran and it conformed to the ethics guidelines of the Declaration of Helsinki. We applied a usability and acceptability testing approach to examine participant's views on the use of Jaga Sehat as a media platform to improve knowledge about healthy living.

One researcher (AI) gave information about the purpose of this pilot program and explained the research procedure, namely: to fill-in online informed consents, to download the mobile app, learn to use it for one day, and to complete an online assessment about the app. The Jaga Sehat app can be downloaded from Google Play. Participants used the app by reading all the material and trying all the features inside it. All participants gave their consent using an online form which included publication of anonymized response. The data was administered via google form and checked.

\section{Instrument}

Data were collected through an online self-reported questionnaire and open-questions which developed by our research group. Usability refers to how well an app functions and whether it serves its intended purpose. ${ }^{24}$ A self-reported questionnaire was used to understand the participant's opinion towards the use of the Jaga Sehat app using Google Form platform. A sociodemographic form asked about the name, student id number, age, email address/phone number and gender. We also wanted to see participant's healthy habits by asking them about their exercise activity ("How often do you exercise?").

The online questionnaire included 11 forced-choice questions, related to content (item 1), language (item 2), illustration (item 3), usability (item 4), layout (item 5), the benefit of information (item 6-9), degree of applicability (item 10) and recommendation for others to use (item 11). All the questions were evaluated using a 5-point Likert Scale from "Strongly Disagree", "Disagree", "Neither Agree nor Disagree", "Agree” to "Strongly Agree”. For question number 
11 about app recommendation, the scale starts from "Never recommend it" to "Definitely recommend it". The total score may range from minimal of 11 and maximal of 55. The score can be obtained by adding up the responses in each item. The Cronbach's alpha of the questionnaire $=0.89$. We used face validity to ensure the validity of the questionnaire. One open-question was added to facilitate suggestions about how to improve and develop the app.

\section{Data Analysis}

Descriptive statistics were used to analyze forced-choice responses and each item was analyzed individually. For the open-ended responses, content analysis was carried out for which descriptive categories were created. The data analysis process started with reading all the answers documents from the participants and built the initial coding directory. One of the authors (AS) built the initial coding directory and provide a transcript code. A discussion was held regularly with the other two authors ( $\mathrm{AI}$ and $\mathrm{AG}$ ) to reach an agreement on the differences in the initial coding. Then, the codes were structured into potential categories to produce a descriptive category. Finally, the discussion was held again to produce a definition and name for each category. These categories were illustrated by typical quotations.

\section{Results}

\section{Demographic Characteristics}

A total of 113 students participated in the current study. The participant's ages ranged from 17 to 22 years old and the majority of them were female (85.8\%). Regarding exercise activity, most of the participants were not exercising regularly and doing it occasionally from time to time. An overview of the demographic data of the study participants is presented in Table 2.

Table 3 shows the means and standard deviations of each survey question. On average, every aspect was considered to be positive, based on participant's responses to every survey question ("agree" or "strongly agree"). Participants reported that Jaga Sehat was well-designed app, functional, and easy to use. The material inside the app was easy to follow with simple and straightforward language. The visual aspects of the app, namely illustration and layout were well received. The app could potentially help them to apply health information and tips in their daily life to maintain their health. The highest score was on the benefit of a positive mindset $(\mathrm{M}=4.47)$, while the lowest was on the app format $(\mathrm{M}=3.95)$.

Table 2 Demographic Characteristics of Study Participants $(\mathrm{N}=\mathrm{I} \mid 3)$

\begin{tabular}{|l|c|c|}
\hline Variables & $\mathbf{N}$ & $\%$ \\
\hline Age (years) & & \\
17 & 28 & $24.8 \%$ \\
18 & 71 & $62.8 \%$ \\
19 & 13 & $11.5 \%$ \\
20 & 1 & $0.9 \%$ \\
\hline Gender & & \\
Female & 97 & $85.8 \%$ \\
Male & 16 & $14.2 \%$ \\
\hline Exercise Activity & & \\
Never & 2 & $1.8 \%$ \\
Rarely & 39 & $34.5 \%$ \\
Sometimes & 65 & $57.5 \%$ \\
Often & 7 & $6.2 \%$ \\
\hline
\end{tabular}

Abbreviations: N, number of participants; \%, frequency. 
Table 3 Participants' Response

\begin{tabular}{|c|c|c|c|}
\hline Aspect & Questions & Mean & SD \\
\hline Content & I. The material on JAGA SEHAT app is easy to understand & 4.35 & 0.60 \\
\hline Language & 2. The material on JAGA SEHAT app use a clear language and easy to understand & 4.46 & 0.62 \\
\hline Illustration & 3. The material on JAGA SEHAT app use illustration and visual explanation that easy to understand & 4.33 & 0.76 \\
\hline Usability & 4. JAGA SEHAT app is easy to operate and use & 4.36 & 0.69 \\
\hline Layout & 5. JAGA SEHAT app is arranged in an interesting format & 3.95 & 0.78 \\
\hline \multirow[t]{4}{*}{ Benefit of information } & 6. The information about healthy diet is beneficial for me & 4.37 & 0.71 \\
\hline & 7. The information about active lifestyle is beneficial for me & 4.44 & 0.65 \\
\hline & 8. The information about positive mindset is beneficial for me & 4.47 & 0.66 \\
\hline & 9. The information about spirituality is beneficial for me & 4.37 & 0.69 \\
\hline $\begin{array}{l}\text { Degree of applicability in } \\
\text { daily life }\end{array}$ & $\begin{array}{l}\text { 10. The information about healthy diet, active lifestyle, positive mindset and spirituality can be } \\
\text { applied in my daily life }\end{array}$ & 4.20 & 0.65 \\
\hline $\begin{array}{l}\text { Recommendation for others } \\
\text { to use }\end{array}$ & II. Would you recommend others to download and use JAGA SEHAT app? & 3.98 & 0.79 \\
\hline
\end{tabular}

Qualitative data were also collected in this survey to compile user feedback about future improvement and development of the app. Suggestions for future improvement and development were divided into five categories: (1) accessibility of mobile app, (2) content, (3) illustration, (4) interactive features, and (5) technical issues.

\section{Availability of Mobile App}

Participants suggested that this app should be available in another operating system besides Android, like iOS. Many of them hoped that this app can be downloaded through the app store so that iPhone users can use it too.

Hopefully it can be developed for Apple users (P.13)

Hopefully it can be downloaded from iOS, so iPhone users can use the app (P.60)

They also supported when this app is distributed among college students and the public at large to make more people use the app.

I hope the app will spread to the wider community (P.6)

\section{Content}

The content referred to every information or material inside the app. Participants said that the material needs to be conveyed in a more detailed manner, for example in tips for a healthy diet, the presenter can be explained about eating schedule. They also emphasized adding more interesting and up-to-date health information. Broadening the scope of material can be a better way to improving the app.

Add more material and the explanation is more detailed, namely the proper eating schedule. The animation can be improved by adding a lot more movement so it will become more interesting (P.23)

Further development can contain more information (P.41)

\section{Illustration}

Illustration referred to the visual aspect of the app. Participants recommended that this app can use more animation on the characters, especially when they have a dialogue with each other. They also proposed applying videography during the presentation of the material. Future improvement can be done by adding a variety of different colors on the characters or the background on each page. Animation on the characters is important because it makes the user feel less bored when 
they read the material. The use of animation in a more sophisticated way may become the app's distinct characteristic that able to attract more people.

Developing better animation, try to add a moving picture in addition to audio, so it will be more interesting. (P.70)

Improving for better presentation and attractive color blend" (P.37)

\section{Interactive Features}

Features category described aspect, quality, or a prominent characteristic that becomes the appeal of an app. Participants expected more interactive features such as behavior tracking, games, and background music. Many mHealth apps provided a set of features related to behavior change for instance tracking, planning, or reminder. For participants, they wanted the app to include a feature that may help them to manage their health like a calorie counter feature, a daily reminder to drink water or exercising and a food planner.

Maybe adding a feature to count calories for a day and reminder feature to remind about exercising schedule. So, the application can be used every day, not only to provide information (P.11)

Participants suggested the use of mini-games and quiz thus there is a mini-game after every part. It served not only for user's entertainment but also helped them checking their understanding of the material. Games are viewed as a distraction to prevent or reduce boredom as they read the materials. They proposed an online counseling feature and Question and Answer feature, with these features they will be able to ask the presenters (health psychologist). Interactive features, namely: games and Question and Answer features are recommended because those features can encourage users to adopt a healthy lifestyle and increasing the retention rate (the chance of user come back to use the app again). Because the app only contained a voice from each character and lack of music, they advised putting background music.

Make it as interesting as possible, so the users won't get bored when listening and reading the materials. Try to make it like a game, so users can check their understanding of the material and won't bore users. For example, you can make few questions about health and the users will answer, and then provide the answer with explanation and animation (P.56)

\section{Technical Issues}

Few technical issues need to be considered. The app must be updated regularly. Future improvements should include more notifications to tell users about various technical problems. For example, a notification that informs the user about the loss of internet connection.

One participant said that he found several bugs inside the app. Aside from that, there is a need to improve consistency between audio and visual component, especially when the characters speak. They also pointed out many typos in the text.

When we open the app, please give notification if internet connection is lost, so we won't be confused if the app suddenly stopped working. Thank you. (P.24)

Please correct all the typos in the text (P.45)

\section{Discussion}

This study aimed to evaluate the usability and acceptability of the Jaga Sehat app. Overall, participants' feedback about using the app were positive. Based on previous studies, people tend to accept mobile apps when the app is well-designed, functional, and easy to use. ${ }^{25,26}$ We believed our app has more value than previous apps concerning the Indonesian culture. First of all, our current healthcare system is heavily influenced by the biomedical approach which focuses on controlling disease and preventing health problems which insufficient for understanding health and illness. ${ }^{27}$ The concept of health and illness are different across cultures and each culture develops its own set of beliefs to explain events related 
to health and illness. By understanding the cultural characteristics of a given group, health promotion programs and services can be customized to better meet the need of its members and increase its receptivity. ${ }^{28}$

Jaga Sehat App has moved beyond a rigid biomedical approach and applied a multidimensional definition of health from WHO. It means putting consideration of the complete person, physically, psychologically, socially, and spiritually in the management of health and prevention of illness. ${ }^{29}$ Because of the influence of culture on how people understanding and perceive health, cultural knowledge is essential to develop appropriate health programs. ${ }^{30}$ Indonesia is an example of a non-Western developing nation with a collectivist culture that emphasizes group membership over and above individual needs and characterized by strong embeddedness of self with family and society. ${ }^{31-33}$ Jaga Sehat delivered a message about the importance of maintaining health and its relation to one's family and social circles which resonate with Indonesian collectivist culture.

Religion/spirituality (R/S) was not included in the original conception of health from WHO, but we introduced it in Jaga Sehat because for many Indonesians religion or spirituality principles become central to everyday life and influence almost all of the life aspects. ${ }^{34}$ Our decision to incorporate the concept of health and R/S in the Indonesian mHealth app was supported by previous research which showed that spirituality is one of the key aspects of well-being among Indonesian people. ${ }^{35}$ Furthermore, in the context of healthcare few studies have shown that Indonesian healthcare providers were more likely to believe R/S influenced patients health, believed it is appropriate to talk and discuss $\mathrm{R} / \mathrm{S}$ with patients and were willing to provide $\mathrm{R} / \mathrm{S}$ support to the patients and their families, ie, by praying together. ${ }^{36-38}$ These results further explained how R/S is embedded in Indonesian people's ideas about well-being and health.

In the Jaga Sehat App, we also cover psychological health as one of the main messages to promote overall health. Previous studies indicated that there is a lack of mental health literacy and a higher stigmatizing attitude towards people with mental illness among Indonesian people. ${ }^{39-41}$ Considering the lack of awareness about mental health in Indonesia, our app can contribute to the further promotion of mental health knowledge. Information regarding psychological health and tips to manage stress can become the first step to increase mental health literacy and effort to preventing stigma and discrimination regarding mental health problems. Better knowledge about mental health was associated with lower stigma toward people with mental illness. ${ }^{42}$ Promoting mental health as inseparable part from other aspects of health is crucial to avoid alienation among people who suffer from mental health problems which subsequently making them less likely to seek help. ${ }^{43}$

Based on the participants comments, they suggested considering the scope of the material and updated information regularly. In developing content for the mHealth app, app developer needs to gain extensive knowledge about the subjects depending on the nature of the app's content demands and user needs. ${ }^{44}$ Participants proposed to add more animation, video, and using a different color scheme to give a distinct characteristic of the app and attracted more people to use it. Aesthetics (layout, graphics, visual appeal) become the second crucial category after functionality to assess the quality of the mHealth app. ${ }^{45}$ For instance, the color was pointed out as a significant visual element regarding beauty, attractiveness, and vividness. ${ }^{46}$

To improve the app, participants proposed to expand the availability of the Jaga Sehat app so it can be operated in many operating systems besides Android, like iOS. After using the app, participants expected more interactive features such as behavior tracking, self-monitoring, reminder, games, mini quiz, online counseling feature, QnA (question and answer), and background music. mHealth apps that provided additional interactive features such as behavior tracking and health status monitoring scored significantly higher in engagement, aesthetics, and overall app quality and can deliver information through interactive and custom-fit content. ${ }^{47,48}$ Because the app only contained a voice from each character and lack of music, they advised putting a piece of background music. These findings implied that there is a need for interactivity while using the app. Using personally engaging and interactive fashion would make users feel involved and enhance the quality of communication in promoting health via technology. ${ }^{49}$

Adding mini-games and quiz is not only to make the app more interesting and attractive, but it also encourages users to check their understanding of the material and reducing boredom as they read the materials. A previous study found that boredom was very persistent across computer-based learning environments and embedding games will be helpful. ${ }^{50}$ The major advantages of integrating gaming experience to bored-ridden activity were ensuring users engagement and 
their focus on health solution and improve user experience with the app by making it into something more enjoyable, competitive, and engaging. ${ }^{51}$ Moreover, interactivity will help users reduced the feelings of boredom and frustration that come from a lack of excitement, which has an adverse effect on technology-based learning.

Several limitations of the current study need to be declared. We used a convenience sampling consisted of dentistry students. A longitudinal dataset across diverse demographic characteristics may be better to represent the public's perceptions and experiences of using the mHealth app and evaluate variation in-app use over time. Different target groups will have different requirements and approaches to digital tools. ${ }^{52}$ Future studies can explore the impact of incentives, social media, gamification and provide the basis for the app developer to incorporate high-quality evidencebased content plus specific change technique to improve the whole aspect of the app. Finally, a larger scale study is needed to evaluate the usability and acceptability of the app in the general population. In the near future, we will improve the mHealth App based on participants suggestion which includes more interactive menu, such as self-monitoring and behavior tracking.

\section{Conclusion}

Our results suggested that there is supporting evidence of the usefulness and acceptability of Jaga Sehat mHealth app. Participants reported that Jaga Sehat was functional, easy to use and have a good design. The language and material were clear and easy to understand. The app could encourage and help them lead a healthy lifestyle; therefore, they would recommend this app for others. Suggestions for future improvement and development were focused on 5 aspects, namely: (1) accessibility of mobile app, (2) content, (3) illustration, (4) interactive features, and (5) technical issues. JAGA SEHAT was considered to have high usability and acceptability.

The implication of the current study finding for daily practice is that the Jaga Sehat mHealth app is a promising educational instrument that can be implemented in Indonesian general population. The preventive approach to health can be a significant step to improve knowledge and encourage individual to implement a healthy lifestyle.

\section{Abbreviations}

APJII, Asosiasi Penyelenggara Jasa Internet Indonesia; iOs, iPhone OS; mHealth, mobile health.

\section{Acknowledgments}

The authors would like to give thanks to all participants who have participated in this study and use the app. The authors would also like to mention Mr. Arie Hadianto Raharjo as visual communication designer and Mr. Eko Fajar Putra as app developer who helped illustrate and design the app. This study was financed by the Ministry of Research, Technology, and Higher Education of the Republic of Indonesia (PTUPT Scheme 2018, contract no. 1462/UN6.I/LT/ 2018).

\section{Disclosure}

The authors report no conflicts of interest for this work. The researchers declare that they do not have any financial interest or benefit that has resulted from the direct applications of this research study.

\section{References}

1. World Health Organization. MHealth: new horizons for health through mobile technologies; 2011.

2. Lupton D. Health promotion in the digital era: a critical commentary. Health Promot Int. 2014;3(1):174-183. doi:10.1093/heapro/dau091

3. Thornton L, Gardner LA, Osman B, et al. A multiple health behavior change, self-monitoring mobile app for adolescents: development and usability study of the Health4Life app. JMIR Form Res. 2021;5(4):e25513. doi:10.2196/25513

4. Torbjørnsen A, Ribu L, Rønnevig M, Grøttland A, Helseth S. Users' acceptability of a mobile application for persons with type 2 diabetes: a qualitative study. BMC Health Serv Res. 2019;19(1):1-14. doi:10.1186/s12913-019-4486-2

5. Kreps GL. Communication technology and health: The advent of eHealth applications. In: Cantoni L, Danowski JA, editors. Communication and Technology. Walter de Gruyter GmbH; 2015:485-496.

6. Bokolo AJ. Application of telemedicine and eHealth technology for clinical services in response to COVID-19 pandemic. Health Technol (Berl). 2021;11(2):359-366. doi:10.1007/s12553-020-00516-4 
7. Bokolo AJ. Integrating telemedicine to support digital health care for the management of COVID-19 pandemic. Int J Healthc Manag. 2021;14 (1):280-289. doi:10.1080/20479700.2020.1870354

8. Research2Guidance. MHealth app developer economics 2016 the current status and trends of the mHealth app market; 2016.

9. Research2Guidance. MHealth app economics 2017/2018 -current status and future trends in mobile health; 2017.

10. Research2Guidance. MHealth app market sizing 2015-2020 data report to size opportunities in the mHealth app market; 2015.

11. Steinhubl SR, Muse ED, Topol EJ. The emerging field of mobile health. Sci Transl Med. 2015;7(283):283rv3-283rv3. doi:10.1126/scitranslmed. aaa3487

12. APJII. Infografis penetrasi \& perilaku penggunaan internet Indonesia survei 2016; 2016.

13. APJII. Infografis penetrasi \& perilaku penggunaan internet Indonesia survei 2017; 2017.

14. APJII. Laporan survei penetrasi \& profil perilaku pengguna internet Indonesia survei 2018; 2018.

15. Kemp S. Digital 2020: Indonesia; 2020.

16. Malvey D, Slovensky DJ. MHealth: Transforming Healthcare. springer; 2014.

17. Rinawan FR, Amelia I, Ardisasmita MN, et al. Understanding mobile application development and implementation to monitor Posyandu data in Indonesia: a 3-year hybrid action research to build " a bridge" from the community to the national scale. Res Sq. 2020. doi:10.21203/rs.3.rs-15584/ v3

18. Octovia LI, Manikam RM, Witjacksono F, et al. mHealth for mother and child health nutrition - a review and proposed design for Indonesia focus on early life nutrition in Indonesia. 2017 th International Conference on Instrumentation, Communications, Information Technology, and Biomedical Engineering (ICICI-BME); 2017:287-290; IEEE.

19. Mangkunegara CN, Azzahro F, Handayani PW. Analysis of factors affecting user's intention in using mobile health application: a case study of halodoc. 2018 International Conference on Advanced Computer Science and Information Systems; 2018:87-92.

20. Salamah Y, Asyifa RD, Afifah TY, Maulana F, Asfarian A. Thymun: smart mobile health platform for the autoimmune community to improve the health and well-being of autoimmune sufferers in Indonesia. 2020 8th International Conference on Information and Communication Technology (ICoICT); 2020:1-6; IEEE.

21. Aisyah DN, Ahmad RA, Artama WT, Adisasmito W. Knowledge, attitudes, and behaviors on utilizing mobile health technology for TB in Indonesia: a Qualitative Pilot Study. Front Public Heal. 2020;8:1-9. doi:10.3389/fpubh.2020.531514

22. Burhan E, Ralena NA, Tahir Y. Improving TB-related knowledge and behavior with Sembuh TB app towards Indonesia TB elimination. Syst Rev Pharm. 2019;10.2(2019):313-317. doi:10.5530/srp.2019.2.42

23. Handayani PW, Meigasari DA, Pinem AA, Hidayanto AN, Ayuningtyas D. Critical success factors for mobile health implementation in Indonesia. Heliyon. 2018;4(11):e00981. doi:10.1016/j.heliyon.2018.e00981

24. Jake-schoffman DE, Silfee VJ, Waring ME, et al. Methods for evaluating the content, usability, and efficacy of commercial mobile health apps. JMIR mHealth uHealth. 2017;5(12):e190. doi:10.2196/mhealth.8758

25. Armstrong S. Which app should I use? BMJ. 2015;351(September):1-3. doi:10.1136/bmj.h4597

26. Mendiola MF, Kalnicki M, Lindenauer S. Valuable features in mobile health apps for patients and consumers: content analysis of apps and user ratings. JMIR mHealth uHealth. 2015;3(2):e40. doi:10.2196/mhealth.4283

27. Berg GV, Sarvimäki A. A holistic-existential approach to health promotion. Scand J Caring Sci. 2003;17(4):384-391. doi:10.1046/j.02839318.2003.00240.x

28. Kreuter MW, Mcclure SM. The role of culture in health communication. Annu Rev Public Health. 2004;25:439-455. doi:10.1146/annurev. publhealth.25.101802.123000

29. Huljev D, Pandak T. Holistic and team approach in health care. Signa Vitae. 2016;11(2):66-69.

30. Winkelman M. Culture and Health: Applying Medical Anthropology. John Wiley \& Sons; 2008.

31. Jaafar JL, Idris MA, Ismuni J, et al. The sources of happiness to the Malaysians and Indonesians: data from a smaller nation. Procedia-Social Behav Sci. 2012;65:549-556. doi:10.1016/j.sbspro.2012.11.164

32. Jetten J, Postmes T, McAuliffe BJ. "We' reall individuals": group norms of individualism and collectivism, levels of identification and identity threat. Eur J Soc Psychol. 2002;32(2):189-207. doi:10.1002/ejsp.65

33. Kitayama S, Markus HR, Kurokawa M. Culture, emotion, and well-being: good feelings in Japan and the United States. Cogn Emot. 2000;14 (1):93-124. doi:10.1080/026999300379003

34. Lippman LH, Keith JD. The demographics of spirituality among youth: international perspectives. In: Roehlkepartain EC, Benson PL, King PE, Wagener LM, editors. The Handbook of Spiritual Development in Childhood and Adolescence. Sage; 2006:109-123.

35. Maulana H, Obst P, Khawaja N. Indonesian perspective of wellbeing: a qualitative study. Qual Rep. 2018;23(12):3136-3152.

36. Herlianita R, Yen M, Chen C-H, Fetzer SJ, Lin EC. Perception of spirituality and spiritual care among Muslim nurses in Indonesia. $J$ Relig Health. 2017;57(2):762-773. doi:10.1007/s10943-017-0437-6

37. Lucchetti G, Ramakrishnan P, Karimah A, et al. Spirituality, religiosity, and health: a comparison of physicians' attitudes in Brazil, India, and Indonesia. Int J Behav Med. 2015;23(1):63-70. doi:10.1007/s12529-015-9491-1

38. Rochmawati E, Wiechula R, Cameron K. Centrality of spirituality/religion in the culture of palliative care service in Indonesia: an ethnographic study. Nurs Health Sci. 2018;20(2):231-237. doi:10.1111/nhs.12407

39. Damayanti R, Hufad A, Kamil M. Stigma, discrimination upon people with mental disorder and mental health literacy in Indonesia. Proceedings of 1st International Conference on Educational Sciences; 2017:398-401. doi:10.5220/0007041803980401.

40. Kristina SA, Mardea NA, Ramadhani F, Aliyah H. Mental health literacy among university students in Yogyakarta. Ment Health (Lond). $2020 ; 25$ (4):2243-2249.

41. Subu MA. Persistent taboo understanding mental illness and stigma among Indonesian adults through grounded theory. 2015.

42. Hartini N, Fardana NA, Ariana AD, Wardana ND. Stigma toward people with mental health problems in Indonesia. Psychol Res Behav Manag. 2018;11:535-541. doi:10.2147/PRBM.S175251

43. Prince M, Patel V, Saxena S, et al. No health without mental health. Lancet. 2007;370(9590):859-877. doi:10.1016/S0140-6736(07)61238-0

44. Bhatheja S, Fuster V, Chamaria S, et al. Developing a mobile application for global cardiovascular education. J Am Coll Cardiol. 2018;72 (20):2518-2527. doi:10.1016/j.jacc.2018.08.2183 
45. Bardus M, Ali A, Demachkieh F, Hamadeh G. Assessing the quality of mobile phone apps for weight management: user-centered study with employees from a Lebanese University. JMIR mHealth uHealth. 2019;7(1):1-10. doi:10.2196/mhealth.9836

46. Silvennoinen J, Vogel M, Kujala S. Experiencing visual usability and aesthetics in two mobile application contexts. J Usability Stud. 2014;10 (1):46-62.

47. Bardus M, van Beurden SB, Smith JR, Abraham C. A review and content analysis of engagement, functionality, aesthetics, information quality, and change techniques in the most popular commercial apps for weight management. Int J Behav Nutr Phys Act. 2016;13(1):1-9. doi:10.1186/s12966016-0359-9

48. Paige SR, Alber JM, Stellefson ML, Krieger JL. Missing the mark for patient engagement: mHealth literacy strategies and behavior change processes in smoking cessation apps. Patient Educ Couns. 2018;101(5):951-955. doi:10.1016/j.pec.2017.11.006.Missing

49. Kreps GL, Neuhauser L. Artificial intelligence and immediacy: designing health communication to personally engage consumers and providers. Patient Educ Couns. 2013;92(2):205-210. doi:10.1016/j.pec.2013.04.014

50. Baker RS, D'Mello SK, Rodrigo MMT, Graesser AC. Better to be frustrated than bored: the incidence, persistence, and impact of learners' cognitive-affective states during interactions with three different computer-based learning environments. Int J Hum Comput Stud. 2010;68 (4):223-241. doi:10.1016/j.ijhcs.2009.12.003

51. Sardi L, Idri A, Fernández-Alemán JL. A systematic review of gamification in e-Health. J Biomed Inform. 2017;71:31-48. doi:10.1016/j. jbi.2017.05.011

52. Brox E, Fernandez-Luque L, Tøllefsen T. Healthy gaming-video game design to promote health. Appl Clin Inform. 2011;2(2):128-142. doi:10.4338/ACI-2010-10-R-0060

Journal of Multidisciplinary Healthcare

\section{Dovepress}

\section{Publish your work in this journal}

The Journal of Multidisciplinary Healthcare is an international, peer-reviewed open-access journal that aims to represent and publish research in healthcare areas delivered by practitioners of different disciplines. This includes studies and reviews conducted by multidisciplinary teams as well as research which evaluates the results or conduct of such teams or healthcare processes in general. The journal covers a very wide range of areas and welcomes submissions from practitioners at all levels, from all over the world. The manuscript management system is completely online and includes a very quick and fair peer-review system. Visit http://www.dovepress.com/testimonials.php to read real quotes from published authors.

Submit your manuscript here: https://www.dovepress.com/journal-of-inflammation-research-journal 